\title{
Caries en una población militar española
}

\author{
Mombiedro Sandoval R. ${ }^{1}$, Llena Puy C. ${ }^{2}$
}

Sanid. mil. 2011; 67 (1): 36-42; ISSN: 1887-8571

\begin{abstract}
RESUMEN:
Objetivo: Evaluar la prevalencia de caries de la población militar española de la base «General Almirante» Marines, Valencia. Material y método. Estudio descriptivo transversal para el que se seleccionó una muestra de 387 militares para un IC del 95\%. En el gabinete dental de la base se realizó la exploración oral siguiendo los criterios de la OMS (1997, 4a edición). Un solo profesional experimentado con un explorador patrón llevó a cabo la encuesta entre diciembre del 2003 y julio del 2004. Resultados. El índice de dientes con caries, ausentes y obturados (CAO-D) fue de 4,05. El 23\% de la muestra estaba libre de caries. Presentaban caries sin tratar el 40\% de los explorados, siendo significativamente superior el porcentaje en la tropa y en los que referían no cepillarse de forma regular. El índice de restauración (\% O/ CAO-D) fue del $60 \%$. La población de tropa presentó con significación estadística mas caries y menos dientes con caries tratada (A,O) que el grupo de oficiales y suboficiales. Conclusiones. Casi la mitad de la población encuestada precisa tratamiento por caries. Estrategias de asistencia basadas en políticas preventivas de diagnostico precoz y de asistencia personalizada en función del riesgo individual de caries, adaptadas al medio militar, pudieran ser la clave para poder desplegar en un futuro tropas que no ocasionen emergencias en futuras operaciones.
\end{abstract}

PALABRAS CLAVE: Caries dental, Epidemiología, Índice DMFT, Personal militar.

\section{Dental caries in a Spanish military population}

SUMMARY:

Object: The target of this study was to assess the prevalence of caries in a population of Spanish military personnel at the «General Almirante» base at Marines (Valencia). Material and Method: A descriptive cross-sectional study was made of a sample of 387 military personnel, (95\% CI). The oral examinations were conducted at the dental clinic at the base, in accordance with the WHO (1997, $4^{\text {th }}$ ed.) criteria, by a single dentist calibrated against an experienced examiner. The survey was conducted between December 2003 and July 2004 . Results: The decayed, missed and filled teeth (DMF-T) were 4.05 . The $23 \%$ of the sample was free of caries. The $40 \%$ of those examined presented untreated caries, the percentage of untreated caries was significantly higher among the privates and among those who did not report regular tooth brushing. The care index ( $\%$ F/DMF-T) was $60 \%$. The privates presented more decay and less treated caries $(\mathrm{M}, \mathrm{F})$ than the officers and non-commissioned officers, these differences were statistically significant. Conclusions: Nearly half of those polled need caries treatment. Strategies based on preventive policy of early diagnosis and Caries management by risk assessment adapted to military life, may be the key to achieving emergency-free troop deployment during future operations.

KEY WORDS: Dental caries, Epidemiology, DMFT index, Military Personnel.

\section{INTRODUCION}

La importancia de la salud general y en especial de la oral, desde el punto de vista militar tiene unas consideraciones y unas repercusiones que le son propias. La pérdida de la salud oral se asocia a repercusiones personales, logísticas y operativas difíciles de cuantificar. La principal causa de morbilidad oral en despliegues y maniobras tanto en nuestro ejército como en los de nuestro entorno, se debieron a la presencia de caries dental, supuso el 55\% de las causas de asistencia en Bosnia-Herzegovina en el año 2000-2001. La segunda causa de asistencias fueron los derivados de problemas gingivales y periodontales (16\%). Un elemento a destacar fue que

${ }^{1}$ Tcol. Estomatólogo. Hospital Central de la Defensa Gómez Ulla. Servicio de Odontología/ Estomatología. Madrid. España.

${ }^{2}$ Universidad de Valencia. Dpto. de Estomatología. Agència Valenciana de Salut Odontólogo de Atención Primaria. Departamento 9. Valencia. España.

Dirección para correspondencia: Rafael Mombiedro-Sandoval. Hospital Central de la Defensa Gómez Ulla. Glorieta del Ejército s/n. Madrid 28047. España. Tel.: 0034914228765. rmomsan@oc.mde.es

Recibido: 11 de febrero de 2009

Aceptado: 3 de septiembre de 2010 la incidencia de asistencias por causas de origen oral de nuestro ejército, supuso entorno al doble que la de los ejércitos americanos e ingleses ${ }^{1}$.

Existirían varias razones que justificarían esta mayor incidencia, unas estarían ligadas a la demografía de la población desplegada (edad, sexo, nivel cultural) otras a las condiciones del despliegue (operaciones de interposición y reconstrucción, combate, facilidad de uso del servicio de salud oral), otros factores estarían relacionados con el concepto de asistencia o de emergencia dental y otros con el estado de salud previo al despliegue, elemento que ha sido el más estudiado $^{2-6}$

El estado de salud oral previo al despliegue en los militares americanos, estaba condicionado por una mayor y mejor utilización de los servicios de salud oral, aspecto que se basa en la instauración de programas de prevención, a una mejor accesibilidad a la asistencia, a la obligatoriedad de un cierto estado de salud oral (aptitud para el despliegue), a la mejora de las ayudas económicas y en general al incremento del nivel cultural sobre la higiene y salud personal, como fue estudiado por Chisick $1995^{7}$, Chisick y cols. $1998^{8}$ y Hyman y cols. $2006^{9}$.

En los países industrializados y también en España, ha mejorado la salud oral en los últimos años, esta mejora de la salud oral en el 


\section{Caries en una población militar española}

mundo industrializado, se debía más que a las técnicas de aplicación individual, a aquellos factores que introducen cambios en el comportamiento de la población y que suponen factores de riesgo, como son: la utilización generalizada de fluoruros, la instauración de servicios preventivos y la mejora en el conocimiento sobre la salud oral de los profesionales y de la población general ${ }^{10-23}$.

La mejoría en la salud oral también se evidencia en los países en desarrollo con planes específicos de prevención de la caries ${ }^{24}$.

Entre los factores que distinguen a la población militar española, y que pudieran actuar como elementos potenciadores de una mejor salud oral, se podría destacar: los controles de salud al ingreso en el ejército, prueba que sus miembros deben superar, en estos reconocimientos se incluye la salud bucal, las ayudas económicas prestadas por el Instituto Social de las Fuerzas Armadas, según la normativa vigente y por la presencia en numerosas Unidades y Bases de gabinetes odontológicos con misiones asistenciales y preventivas sin coste directo al usuario ${ }^{25-27}$.

El estado de salud oral previo al despliegue de los militares españoles, estuvo determinado por las exploraciones bucales previas a las misiones fuera del territorio nacional. Estas se comenzaron a realizar, sobre los años noventa, durante nuestra intervención en la antigua Yugoslavia. Las exámenes orales se realizaban, en la llamada "fase de concentración» en los días previos a la misión, se realizaba en ocasiones en habitaciones o locales multiuso, situación que no permitía la normalización de la exploraciones. Los resultados, se comunicaban al interesado y al los Jefes de Unidad, por falta de desarrollo normativo, no presentaban repercusiones para el servicio ni para el despliegue.

Con el protocolo de 07/01 de 2005 de la Instrucción Técnica 01/03 de 10 de julio de 2003 de la Inspección General de Sanidad (IGESAN) se regularizó la exploración. Se sistematizaron los procedimientos, donde y cuando, también se determinó el material y el método exploratorio a seguir. En esta instrucción técnica, se consideró «apto para el despliegue» o «despleglable» a todo militar cuyo estado bucal no requiera asistencia o que sea improbable que pueda necesitar tratamiento dental de emergencia en los próximos 12 me$\operatorname{ses}^{28,29}$, también se aprobó la ficha odontológica a cumplimentar. La exploración dental se hizo preceptiva en los meses previos al despliegue, para dar tiempo a que se pudieran tratar las enfermedades o situaciones con suficiente antelación. Este protocolo fue derogado por la Instrucción Técnica de la IGESAN de 26/07 de 2006, «Instrucción Técnica sobre reconocimiento odontológico básico y formalización de la ficha dental», por la que se adopta la ficha dental empleada para la identificación de la Instrucción técnica de 7 de febrero de 2006 de la Inspección General de Sanidad (IGESAN), sobre la ficha de identificación sanitaria (FIS) y huella genética (0702-2006). La última instrucción técnica de la IGESAN dirigida a los requisitos sanitarios exigibles al personal que se desplace a zonas de operaciones, que trata la salud dental predespliegue, está fechada el 5 de febrero de 2009, en ella se mantienen los criterios y la documentación anteriormente aprobada.

En el momento actual no se dispone de información del estado de salud oral de la población militar española y más concretamente de la experiencia de caries, por lo que en el presente estudio nos propusimos analizar la experiencia de caries, de una muestra de la población militar de la Base General Almirante de Marines de Valencia. Las ventajas de conocer la salud oral y determinar las necesidades de tratamiento, es de gran relevancia por su finalidad pre- ventiva y previsora. Por un lado se pueden buscar grupos de riesgo, establecer prioridades y poder en un futuro proyectar planes de salud oral específicos. Por otro lado para poder desplegar con los medios adecuados a las necesidades, adaptándose lo más posible a los principios logísticos generales ${ }^{30,31}$, se necesitaría prever el número y la naturaleza de emergencias dentales en operaciones, tan importante es conocer que materiales e instrumentos serán necesarios como el perfil del profesional a desplazar ${ }^{31,32}$.

\section{MATERIAL Y MÉTODOS}

Se diseño un estudio exploratorio observacional transversal oral con arreglo a los criterios OMS/WHO de $1997^{33}$.

La muestra estuvo compuesta por 387 militares, pertenecientes a la Base General Almirante de Marines de Valencia. El cálculo del tamaño muestral se realizó para un intervalo de confianza del $95 \%$, tomando como referencia, los índices de caries del grupo de población de 35 a 45 años del estudio epidemiológico nacional del año 2000.

Se utilizó un muestreo sistemático de los sujetos que acudían al Botiquín de la Unidad, por alguna de las siguientes razones: Reconocimiento de conductores, exámenes periódicos en salud, tareas periciales como el seguimiento de bajas, pacientes con pequeñas lesiones, vacunaciones y necesidades administrativas. La asistencia al botiquín no supone necesariamente que el sujeto padezca una enfermedad. Los criterios de inclusión fueron el ser militar y estar destinado en la Base General Almirante de Marines. Los criterios de exclusión fueron padecer alguna enfermedad o dolencia que produjera afectación o deterioro del estado general, o que asistieran a la consulta por presentar odontalgia (ocho pacientes).

A todos los explorados se les solicitó consentimiento informado, los sujetos explorados fueron informados de las condiciones de salud en la que se encontraban, y se le propusieron medidas preventivas y terapéuticas específicas a su condición de salud. En caso de requerir asistencia, se les proporcionó en el momento o se derivaron a los servicios odontológicos que le correspondieran por su filiación a una compañía privada de seguro.

La recogida de datos se realizó por un solo explorador, se inició en diciembre del 2003 y se finalizaron en julio del 2004. Las exploraciones se realizaron en el gabinete odontológico del botiquín de la Base Militar, utilizando un espejo bucal plano del número 5, una sonda periodontal tipo OMS (PCP 11 5B) y de la unidad dental se utilizó la pistola de aire a presión para secar la superficie de los dientes y eliminar los restos más groseros y el foco del equipo como fuente de luz. Los criterios para la valoración de la caries fueron los propuestos en el manual de la OMS para estudios epidemiológicos de $1997^{33}$.

Otras variables que se recogieron fueron la edad, el sexo y el empleo militar de los encuestados, la frecuencia de cepillado y el hábito tabáquico.

Para asegurar la fiabilidad y validez de los resultados el explorador fue calibrado inicialmente con un explorador experimentado, obteniéndose un índice Kappa de 0,85 y un porcentaje de acuerdo del 99,4\%. Así mismo para asegurar la consistencia a lo largo del estudio, el explorador duplicó 38 exploraciones (10\%) y se efectuó el cálculo de la concordancia intraobservador que fue del 99\% y del índice Kappa que fue de 0,83.

Para el análisis estadístico se empleó el paquete SPSS. 15.0 (SPSS Inc., Chicago, IL). Se realizó un análisis descriptivo em- 
Tabla 1. Distribución de la muestra por edades (años), sexos y empleo.

\begin{tabular}{|lccccccccc|}
\hline & \multicolumn{3}{c}{ Varones } & \multicolumn{3}{c|}{ Mujeres } & \multicolumn{2}{c|}{ Totales } \\
\hline & Frecuencias & Edad Media & $\mathbf{p}$ & Frecuencias & Edad Media & p & Frecuencias & Edad Media & p \\
\hline Tropa & $213(72,4 \%)$ & $23,6(3,77)$ & & $81(27,6 \%)$ & $23,8(3,3)$ & & $294(76 \%)$ & $23,7(3,65)$ & \\
O/So & $89(95,7 \%)$ & $39,3(7,3)$ & $<^{\prime} 001^{*}$ & $4(4,3 \%)$ & $33,2(5,1)$ & $<^{\prime} 001^{*}$ & $93(24 \%)$ & $39,04(7,4)$ &, $000^{*}$ \\
\hline Totales & $302(78 \%)$ & $28,8(8,78)$ & $<^{\prime} 001^{*}$ & $85(22 \%)$ & $24,28(3,9)$ & $<^{\prime} 001^{*}$ & $387(100 \%)$ & $27,39(8,1)$ & \\
\hline
\end{tabular}

* $\mathrm{p}<0,05$ U de Mann Whitney.

O/So. Oficial/Suboficial.

pleando como medidas de tendencia central la media aritmética y de dispersión la desviación típica para las variables cuantitativas; para las variables categóricas se empleó como índice descriptivo la frecuencia absoluta y relativa en porcentajes. Como medida de asociación se empleó dependiendo del tipo de variables a comparar, para las variables categóricas o cualitativas, el test Chi cuadrado de Pearson, y ante la falta de normalidad de los valores cuantitativos o numéricos se utilizó el test de U de Mann-Whitney. Se realizó una Regresión Logística utilizando como variable dependiente la presencia de caries sin tratar dicotomizada $(0=$ no caries $\sin$ tratar, $1=$ caries sin tratar) y como independientes la edad, el sexo, el tipo de empleo y la frecuencia de cepillado, calculándose la Odds Ratio. El intervalo de confianza empleado fue al $95 \%$, considerándose como estadísticamente significativo un valor de la $\mathrm{p}<0,05$.

\section{RESULTADOS}

La población encuestada estaba compuesta en cuanto al empleo militar se refiere, por un $76 \%$ (294 personas) de tropa y un $24 \%$ (93 personas) de oficiales y suboficiales $(\mathrm{O} / \mathrm{So})$, respecto al sexo, el $78 \%$ (302 personas) eran varones y el 22\% (85 personas) mujeres. Para ambas variables las diferencias fueron estadísticamente significativas. Las edades de los sujetos de la muestra estaban en un rango comprendido entre los 18 y los 53 años, la mediana fue de
24 años, y la media de 27,4 (8,1). Más datos pueden ser observados en la Tabla 1.

Se valoraron los índices de caries (C), ausentes (A) y obturados (O) por diente (CAO-D) también se revisaron los índices de restauración (IR), y el porcentaje de pacientes libres de caries, los resultados se muestran en la Tabla 2.

La población encuestada presentó un índice CAO-D de 4,05, casi el $60 \%$ del índice lo ocuparon los dientes obturados, la media de dientes ausentes y cariados fueron 0,87 y 0,79 respectivamente. El 23\% de los encuestados se encontraban libres de caries y la presencia de lesiones se concentró en el 40,1\% de la muestra.

En el análisis por edades, encontramos que el grupo de mayor edad, presentó una mayor experiencia de caries, con mayor CAO-D que los más jóvenes, esta mayor experiencia de caries se debió sobretodo a la mayor presencia de caries tratada (A y O).

Analizando los resultados por el tipo de empleo militar, observamos que las diferencias se caracterizaron en que la tropa presentó menores índices CAO-D que los $\mathrm{O} / \mathrm{So}$, pero concentraba la mayoría de la necesidad de tratamiento por caries (45\%). Los O/So presentaron mas caries tratada que la tropa.

Por sexos, las mujeres presentaron mayor experiencia de caries que los hombres, los mayores índices CAO-D lo fueron por la mayor presencia de dientes obturados y con caries.

Los sujetos que decían cepillarse los dientes todos los días, mostraron mayores índices CAO-D pero menos necesidad de trata-

Tabla 2. Índices distribuidos por grupos de edad, empleo militar, sexo, cepillado diario y fumador habitual.

\begin{tabular}{|c|c|c|c|c|c|c|c|c|c|}
\hline & & $\mathbf{N}^{\mathbf{o}}$ & $\mathrm{C}$ & A & $\mathbf{O}$ & IR & CAO-D & Sin Caries & $\mathrm{C}>0$ \\
\hline \multirow{3}{*}{ EDAD } & $<25$ años & 186 & $0,82(1,23)$ & $0,32(0,72)$ & $1,52(2,33)$ & $57,14 \%$ & $2,66(2,35)$ & $29,6 \%$ & $41,9 \%$ \\
\hline & $\geq 25$ años & 201 & $0,75(1,28)$ & $1,38(2,18)$ & $3,21(3,42)$ & $60,11 \%$ & $5,34(4,57)$ & $16,9 \%$ & $38,3 \%$ \\
\hline & $\mathbf{p}$ & & & $<^{`} 001 *$ & $<^{\prime} 001 *$ & & $<^{\prime} 001 *$ & '004* & \\
\hline \multirow{3}{*}{ EMPLEO } & Tropa & 294 & $0,92(1,36)$ & $0,60(1,38)$ & $1,98(2,70)$ & $56,4 \%$ & $3,51(3,59)$ & $23,8 \%$ & $44,6 \%$ \\
\hline & O/SO & 93 & $0,35(0,69)$ & $1,71(2,36)$ & $3,71(3,60)$ & $64,3 \%$ & $5,77(4,81)$ & $20,4 \%$ & $25,8 \%$ \\
\hline & $\mathbf{p}$ & & $<^{\prime} 001 *$ & $<^{‘} 001 *$ & $<^{\prime} 001 *$ & '014* & $<^{‘} 001 *$ & & '002* \\
\hline \multirow{3}{*}{ SEXO } & Varón & 302 & $0,71(1,18)$ & $0,87(1,68)$ & $2,09(2,95)$ & $56,94 \%$ & $3,67(3,99)$ & $26,5 \%$ & $38,1 \%$ \\
\hline & Mujer & 85 & $1,05(1,45)^{*}$ & $0,87(1,91)$ & $3,51(3,03)$ & $64,76 \%$ & $5,42(3,87)$ & $10,6 \%$ & $47,1 \%$ \\
\hline & $\mathbf{p}$ & & '029* & & $<001 *$ & '044* & $<^{\prime} 001^{*}$ & $<002 *$ & \\
\hline \multirow{3}{*}{ DIARIO } & No & 140 & $1,0(1,44)$ & $0,60(1,28)$ & $1,81(2,62)$ & $53,07 \%$ & $3,41(3,29)$ & $19,4 \%$ & $47,5 \%$ \\
\hline & SI & 247 & $0,66(1,12)$ & $1,01(1,93)$ & $2,70(3,16)$ & $61,78 \%$ & $4,37(4,32)$ & $25,1 \%$ & $35,9 \%$ \\
\hline & $\mathbf{p}$ & & '040* & '041* & $<^{\prime} 001^{*}$ & '013* & '004* & & '031* \\
\hline \multirow{3}{*}{$\begin{array}{l}\text { FUMADOR } \\
\text { HABITUAL }\end{array}$} & No & 182 & $0,70(1,14)$ & $0,87(1,87)$ & $2,73(3,21)$ & $63,48 \%$ & $4,30(4,08)$ & $19,8 \%$ & $38,5 \%$ \\
\hline & SI & 205 & $0,86(1,34)$ & $0,87(1,61)$ & $2,10(2,83)$ & $54,80 \%$ & $3,83(3,98)$ & $25,9 \%$ & $41,5 \%$ \\
\hline & $\mathbf{p}$ & & & & '041* & & & & \\
\hline TOTAL & & 387 & $0,79(1,25)$ & $0,87(1,73)$ & $2,40(3,03)$ & $59,25 \%$ & $4,05(4,03)$ & $23,0 \%$ & $40,1 \%$ \\
\hline
\end{tabular}

$\mathrm{C}=$ Caries; $\mathrm{A}=$ Ausentes; $\mathrm{O}=$ Obturaciones (media y desviación típica); $\mathrm{IR}=$ Índice de restauración $=\mathrm{O} \times 100 / \mathrm{CAO}-\mathrm{D} ; \mathrm{C}>0=\%$ de encuestados con caries; $\mathrm{O} / \mathrm{SO}=\mathrm{O}$ * $\mathrm{p}<0,05$, U de Mann-Whitney, y $\chi 2$. 
miento de caries que los que no se cepillaban con regularidad. Los mayores índices CAO de los que se cepillaban a diario, se debió a la a la mayor presencia de caries tratada.

El hábito tabáquico no mostró asociación con la presencia de caries, en los no fumadores se observó un mayor componente de dientes obturados frente a los que fumaban, pero no influyo en la experiencia de caries con significación estadística.

Las necesidades de tratamiento por caries en nuestra población militar, fue de casi una caries por individuo, supuso que el 19,5\% de la patología se encontraba sin tratar. Esta necesidad se concentró en el $40 \%$ de la población estudiada, más en la tropa en las mujeres y en los que no se cepillan a diario que en los O/So los hombres o los que si se cepillaban diariamente.

Se analizó mediante regresión logística múltiple la variable presencia de caries o caries sin tratar dicotomizada $(0=$ no caries $\sin$ tratar, $1=$ caries sin tratar), utilizando como variables explicativas el tipo de empleo, cepillado diario, sexo y cepillado por empleo. De las variables estudiadas, el modelo definitivo recoge únicamente la variable empleo como variable explicativa de las diferencias en la presencia de caries. El efecto que sobre la presencia de caries manifestó la tropa fue de una Odds Ratio (OR) 2,3 (IC95\%: 1,37-3,88) superior a la que presentaron $\operatorname{los} \mathrm{O} / \mathrm{So}(\mathrm{p}=0,002)$. El cepillado diario por empleo, el cepillado, el sexo, y la edad, se extrajeron del modelo matemático sin cambio significativo de la verosimilitud del mismo.

\section{DISCUSIÓN}

La comparación de nuestros resultados con otras encuestas nacionales e internacionales, se ve dificultada por varios factores, uno de ellos es el ligado al método exploratorio empleado, en otros casos las dificultades radican en las diferentes cohortes etarias y culturales estudiadas, diferentes a la población general española.

En cuanto al método exploratorio intervendrían por un lado, las propias condiciones de la exploración: Cuando las lesiones están cavitadas y se presentaban en dientes limpios y secos y se dispone de la adecuada iluminación, con solo la inspección ocular ayudado con una sonda roma, las caries de localizaron oclusal eran de fácil diagnóstico, con sensibilidades entre el $97 \%$ y el $85 \%$. En las lesiones proximales, con solo la exploración clínica, se alcanzaban sensibilidades del 70\% al 80\%. Estos sistemas OMS/WHO de exploración recomendados para poblaciones, tienen como ventaja, la alta especificidad, en torno al $95 \%{ }^{34-38}$.

El bajo índice CAO-D de nuestro trabajo, puede ser consecuencia de la tendencia mostrada a la disminución en la prevalencia de caries encontrada en el mundo industrializado, tanto en la población civil como en la militar.

El índice CAO-D de nuestra población de 27 años de edad promedio $(4,05)$, se asemejó más a los resultados obtenidos en las encuestas a estudiantes de odontología $(5,2)$ y medicina $(3,3)$ del año $1994^{39}$, que a los índices de población adulta joven española (35-44 años) de los años $2000^{16}$ y $2005^{17}$ (8,4 y 9,6 respectivamente). Los índices de nuestra población que más se acercaron a los de la población general adulta joven española fueron los correspondientes a los de las cohortes de mayores de 25 años y la de los $\mathrm{O} / \mathrm{So}$, (5,34 y 5,77 respectivamente).

Nuestra población de militares, presentaba mejor índice por tener menos caries, menos ausentes y menos obturados que la población general española de 35 a 45 años de los años $2000^{16}$ y $2005^{17}$.
El índice CAO-D de la población de tropa de nuestro estudio $(3,5)$ fue semejante a la de soldados de reemplazo del año $1995^{40}$ $(4,0)$, pero si analizamos la composición del índice, en nuestra encuesta predominó el componente de obturación (1,98-60\%) y en el año 1995 prevaleció el componente de caries $(2,58-63,2 \%)^{40}$.

Los índices CAO-D de nuestra población militar $(4,0)$, se asemejaron a los encontrados en los últimos años en población de militares en Australia $(6,8)^{41},(4,6)^{41},(3,4)^{42}$; Italia $(3,6)^{43}$; y Brasil $(5,7)^{44}$, $(4,5)^{45},(4,6)^{46}$, y ligeramente inferior a los índices de militares de Turquía $(5,9)^{47}$ e Israelíes $2004(6,77)^{48}$.

La comparación con los militares de América de $1966^{49}$ y de Gran Bretaña $1980^{50}, 1988^{51}$ y $1992^{51}$, se ve dificultada por la diferencia metodológica en la exploración, y por los años trascurridos. No obstante, todos ellos mostraron superiores índices CAO-D a los nuestros e índices de restauración semejantes o mejores que los de nuestra investigación.

La población militar de Australia ${ }^{42}$, de Gran Bretaña ${ }^{50}$ e Israel ${ }^{52}$ presentaron mejor salud dental que las mismas cohortes etarias de civiles, con menos caries, más dientes obturados y menos ausentes. En cuanto al edentulismo, este era muy infrecuente en la población joven, pero más infrecuente todavía en los militares de Gran Bretaña y de Israel, que en su contraparte civil ${ }^{50,52}$.

En el ejército, los oficiales representan, frente tropa, el grupo con mayor nivel de escolaridad, a pesar de la dificultad de comparar dos cohortes de edades diferentes, en nuestra encuesta como en el ejercito Israelí, estos últimos suelen presentar menos obturaciones y más caries ${ }^{53}$. La importancia del nivel cultural del soldado y recluta también se estudió en Brasil por Peres M.A. y cols en 2005, observándose que el nivel cultural personal y materno tenía más importancia para la salud dental del joven recluta de 19 años que el nivel cultural paterno y que el nivel económico familiar ${ }^{44}$.

En nuestra encuesta como en la de militares Israelíes ${ }^{48,52-54}$ e italianos $^{43}$ los militares de mayor empleo, presentaron menos caries sin tratar, y más obturaciones. En varias encuestas las mujeres militares ó civiles presentaron mayor índice CAO-D a expensas del componente $\mathrm{O}^{52,54,55}$, también se ha observado en la población general adulta joven española ${ }^{16,17}$, en nuestra encuesta se corrobora este hecho.

En los militares israelíes, el índice CAO-D fue más elevado con más caries sin tratar y más ausentes en el grupo de encuestados que se caracterizaban por: ser fumadores, tener una irregular higiene dental, tener cuatro ó más hermanos, periodo de escolarización corto o ser emigrante de países en desarrollo ó de la antigua Unión Soviética ${ }^{53}$.

El índice de restauración (IR), no ha dejado de ascender en la población general joven española de 35 a 44 años; así del 13\% del año $1983^{14}$ pasó al $27 \%$ en el año $1993^{15}$, y a cerca del $50 \%$ en el $2000^{16}$. Alcanzándose el $54 \%$ en la encuesta del año $2005^{17}$. En nuestro trabajo, el IR sobrepasó el 60\%, mayor en los O/So que en la tropa. Este índice de restauración alto fue más semejante a los resultados de los años noventa en estudiantes Universitarios de estomatología, odontología y medicina ${ }^{39,56,57}$.

El grupo con el menor IR de nuestra encuesta fue el de tropa (56\%), porcentaje alto si se compara con los resultados obtenidos en los trabajadores de Sevilla $1989^{58}(10,7 \%)$, o en los soldados de reemplazo de los años noventa (19\% a 33\% $)^{40,58-60}$.

La comparación de los resultados con los obtenidos a los militares israelíes ${ }^{48,53,54}$, militares americanos del $1977^{49}$, ingleses de los años 1988 y $1992^{51}$ y de los militares de los países escandinavos ${ }^{61-69}$, aunque difícilmente comparables por su diferente metodología, 
mostraron superiores índices CAO-D a los nuestros e índices de restauración semejantes o superiores a los de nuestra encuesta.

La comparación de cohortes de militares frente a las de civiles se ve dificultada por el sistema de exploración, cohorte etaria y año de la encuesta. Aún así, se puede observar, que en nuestra encuesta como en Gran Bretaña e Israel los militares tenían menos porcentaje de edéntulos y mayor índice de restauración que los civiles ${ }^{50,70-72}$. En Estados Unidos los militares presentaron menos caries sin tratar y mayor IR que los civiles ${ }^{73}$. En Australia los militares presentaron menor CAO-D, que los civiles ${ }^{41,42,74}$.

En nuestra encuesta como en el ejército Israelí, los no fumadores y los que se cepillaban los dientes diariamente presentaron mas caries tratada, nuevos estudios serian necesarios para comprender las causas de este mayor índice de tratamiento ${ }^{53}$.

$\mathrm{Al}$ contrario que Sgan-Cohen ${ }^{54}$, Aleksejuniene ${ }^{75}$ en nuestra encuesta el hábito de fumar no se relacionó con mas experiencia de caries, aunque si con un menor índice de restauración.

Con respecto a la experiencia de caries, en nuestra encuesta como en todas las encuestas en población general española consultadas, se apreció un empeoramiento de los índices con la edad. Así, se encontraron más caries tratadas, es decir, más dientes ausentes, más obturaciones y en general mayor CAO-D con significación estadística en las cohortes de más edad ${ }^{15-17}$.

La experiencia de caries en nuestra población fue del 87\%, aún siendo un porcentaje alto, fue inferior que el de la población general española de 35 a 44 años que no bajó del 96,4\% entre los años 1983 al $2005^{14-17}$.

Se observó que la experiencia de caries de la población de tropa de nuestra población (23 años de media) fue del 76\%, menor que los datos obtenidos por la tropa de reemplazo de los años noventa $(85-88 \%)^{40,61}$. Los soldados libres de caries han pasado del $14 \%$ al $23 \%$ en nueve años desde el 1995 al 2004, según los datos de nuestra encuesta ${ }^{40}$.

En nuestra encuesta, el promedio de caries activa, fue del $40 \%$, ascendiendo al $44,6 \%$ en la tropa y mejorando al $25,8 \%$ en los $\mathrm{O} / \mathrm{So}$. El porcentaje de población general española adulta joven con caries activa mejoró desde el año 1993, al año 2005, pasando del 80,7\% al 50,6\%. Descensos que no llegaron a los porcentajes de nuestra encuesta $^{15-17}$. En los soldados de reemplazo de los años 1993-1994, la presencia de caries activa fue de $77,3 \%$; casi el doble que en la población de tropa de nuestro estudio ${ }^{59}$.

Parece, que la tendencia era que la presencia de caries, se concentrase en un núcleo de la población, que estaba por encima de un tercio de la población encuestada. Esta población en riesgo de presentar una emergencia dental por causa de la caries, se concentró en la población de tropa con un riesgo doble (OR 2,3) al de los O/So.

El porcentaje de personas que necesitaba asistencia por caries ha descendido en la población adulta joven española de los últimos años. Desde el $80 \%$ de la población con necesidad de asistencia en el año $1983^{14}$ y $1993^{15}$, pasó al $50 \%$ en los años $2000^{16}$ y $2005^{17}$. En nuestra encuesta se encontró en el $40 \%$ de la población encuestada, más en la tropa que en los $\mathrm{O} / \mathrm{So}$.

La tendencia a la reducción en la necesidad de asistencia en la cohorte de 35 a 44 años manifestadas en las encuestas nacionales de los años 1983, 1993, 2000 y 2005, siguió manifestándose en nuestro estudio. De un índice promedio de necesidad de 4,0 por persona en el año $1983^{14}$, descendió a 2,53 por persona en el año $1993^{12}$ y a 1,50 por persona en el $2000^{16}$ y $2005^{17}$. Este índice en nuestra encuesta fue de 0,79 por persona, más en la tropa que en los O/So.
La asociación de cepillado irregular y caries ha sido evidenciada en diversos estudios ${ }^{76}$, en nuestra encuesta, las personas que se cepillaban de forma irregular, se caracterizaban por presentar mas necesidad de tratamiento de caries que la población que se cepillaba al menos una vez al día.

Las más actuales tendencias en los uniformados americanos surgieron del Departamento de Defensa Americano responsable del sistema de salud militar, que junto con el Tri-Service Center for oral Health Studies, hicieron una propuesta en el 2002. Se pretendía clasificar al personal uniformado en función del riesgo de presentar una emergencia oral, en riesgo bajo, medio y alto. Se presentó una lista de circunstancias que nos indicarían en que riesgo se encuentra el paciente, también se estableció una lista con las actitudes terapéuticas para cada nivel de riesgo. Esta filosofía de diagnóstico y tratamiento se basa en las recomendaciones de la American Dental Association (ADA) del $1995^{77,78}$ y en las técnicas CAMBRA (Caries management by risk assessment) ${ }^{79}$.

Bajo estos criterios, se perseguía clasificar a los militares americanos según nivel de riesgo de caries en bajo, medio y alto. Los pacientes con «riesgo de caries bajo», serian aquellos que no han tenido caries en los pasados tres años, las superficies restauradas lo están de forma adecuada y las fosas y fisuras están selladas o son poco profundas, tiene buena higiene oral y las visitas periódicas al dentista han sido regulares. En situación de «riesgo de caries alto» se clasifica a aquellas personas con: caries presente, las restauraciones tienen márgenes abiertos o desbordados, hay caries de raíz tratada, las fosas y fisuras son estrechas y profundas, las manchas blancas están generalizadas, se aprecian lesiones de radiolucidez interproximal, la higiene oral es mala y no usa o hace uso inadecuado del flúor. Las situaciones de riesgo medio serian situaciones intermedias ${ }^{77-82}$.

Otros factores de riesgo contemplados que clasificarían al paciente el grupo de «riesgo alto de caries» serían: frecuente consumo de azúcares (más de tres bebidas o comidas azucaradas al día), altos títulos de bacterias cariogénicas, bajo flujo de saliva, prolongada lactancia, familia con antecedentes de mala salud, presencia de displasias del esmalte, anormalidades genéticas en los dientes, muchas superficies restauradas, haber sufrido tratamiento con radio o quimioterapia, presentar desórdenes de la alimentación, y consumir alcohol o drogas de abuso ${ }^{77-83}$.

En un trabajo sobre 66.484 soldados americanos, una persona de «Alto riesgo de caries» supuso 15,6 veces más probable necesitar asistencia dental que los de bajo riesgo ${ }^{81}$.

Estos modelos predictivos revisados, están sujetos a la prevalencia de caries y a las características demográficas de la población estudiada, y por lo tanto para otras prevalencias y otras poblaciones debieran ser reevaluados y validados ${ }^{81,82,84}$.

Los protocolos terapéuticos se salen de los objetivos de esta revisión, únicamente comentar que la prevención del tabaquismo y el consejo de nutrición, se encontraban incluidos dentro del capítulo de tratamiento. En estos protocolos, el odontólogo participa como un miembro más del equipo multidisciplinario de salud integral, tratando y manejando situaciones o enfermedades que afectan a la disponibilidad o aptitud para el servicio del militar ${ }^{80}$.

El paciente una vez diagnosticado y tratado con arreglo a su nivel de riesgo de caries, se le motivaba sin amenazas o medidas coercitivas, porque el fin último es modificar los comportamientos de salud y conseguir disminuir el riesgo de caries. Al paciente hay que motivarle con el objetivo de que sea capaz de mantenerse en salud ${ }^{77-84}$. 


\section{Caries en una población militar española}

\section{CONCLUSIONES}

En España la población general y la población militar en particular, no han sido ajenas a la mejora de la salud oral tal como sucede en el mundo industrializado.

La accesibilidad a los servicios sanitarios y la motivación personal, son dos elementos que pueden haber modulado un índice de restauración alto en nuestra población de estudio, no obstante, se mantiene un grupo poblacional con necesidad de asistencia.

El despliegue a la zona de operaciones de un militar con procesos odontológicos agudos supone una baja y una necesidad de asistencia segura. Para que en un futuro se puedan desplegar tropas sin riesgo de ocasionar emergencias previsibles durante el despliegue o maniobra, podría ser conveniente la planificación e implantación de una política preventiva y asistencial de salud buco dental personalizada en función del riesgo individual y adaptada al medio militar, definiendo recursos, objetivos y prioridades. Se necesitaría establecer los sistemas de vigilancia buscando tendencias y grupos de riesgo donde se acumulan las necesidades de tratamiento. La incorporación al ejército de ciudadanos de otras culturas, pueden ser el origen de cambios de tendencias en la salud oral.

El mantenimiento de la salud oral además de las repercusiones para el servicio, puede suponer un incentivo en el mantenimiento del compromiso de los militares profesionales, por otro lado, es un elemento que contribuye a una mayor presencia de la Sanidad Militar en el apoyo a la Fuerza.

\section{AGRADECIMIENTOS}

Al General de Brigada Sub Inspector de Sanidad D. Francisco J. Domingo Gutiérrez, y a todos los militares destinados en la Base «General Almirante» por el apoyo prestado, sin ellos este trabajo no se hubiera podido llevar a cabo.

\section{BIBLIOGRAFÍA}

1. Mombiedro R, Llena C. Emergencias orales en los militares españoles desplegados en Bosnia Herzegovina. Incidencias durante 9 semanas (2000-2001). RCOE 2007; 12: 237-244.

2. Chisick MC, King J. Dental epidemiology of military operations. Mil Med 1993 158: 581-585.

3. Chaffin J, King JE, Fretwell LD. US Army dental emergency rates in Bosnia. Mil Med 2001; 166: 1074-1078.

4. Alexander DCC. Dental recall status and unscheduled dental attendances in British warships. Mil Med 1996; 161: 268-272.

5. Richardson PS. Dental morbidity in United Kingdom Armed forces Iraq 2003. Mil Med 2005; 170: 536-554.

6. Dunn WJ, Langsten RE, Flores S, Fandell JE. Dental emergency rates at two expeditionary medical support facilities supporting Operations enduring and Iraqi freedom. Mil Med 2004; 169: 510-514.

7. Chisick MC. Comparing dental utilization of US Army soldiers with their employed civilian cohorts. Community Dent Oral Epidemiol 1995; 23: 222-225.

8. Chisick MC, Poindexter FR, York AK. Comparing dental utilization rates of active duty US military personnel and their employed civilian cohorts. Mil Med 1998; 163: 148-150.

9. Hyman JJ, Reid BC, Mongeau SW, York AK. The military oral health care system as a model for eliminating disparities in oral health. J Am Dent Assoc 2006; 137 : 372-378.

10. Pieper K, Schulte AG. The decline in dental caries among 12-year-old children in Germany between 1994 and 2000. Community Dent Health 2004; 21: 199-206.
11. Beltrán-Aguilar E, Estupinan-Day S, Baez R. Análysis of prevalence and trends of dental caries in the America between the 1970s and 1990s. Int Dent J 1999; 49: 322-329.

12. Beltrán-Aguilar ED, Barker LK, Canto MT, Dye BA, Gooch BF, Griffin SO, Hyman J, Jaramillo F, Kingman A, Nowjack-Raymer R, Selwitz RH, Wu T. Surveillance for dental caries, dental sealants, tooth retention, edentulism, and enamel fluorosis- United States, 1988-1994 and 1999-2002. MMWR Surveill Summ 2005; 26: 1-43.

13. Gimeno de Sande A, Sánchez B, Viñez J, Gómez F, Mariño F. Estudio epidemiológico de la caries dental y patología bucal en España. Rev Sanid Hig Pública Madrid 1971; 45: 361-433.

14. Cuenca E. La encuesta de la OMS sobre la salud buco dental en España. Una aproximación personal. Arch Odontoestomatol 1986; 2: 15-22.

15. Noguerol-Rodríguez B, Llodra-Calvo JC, Sicilia-Felechosa A, Follaza-Murcia M. La salud bucodental en España 1994. Antecedentes y perspectivas de futuro. Madrid: Ed Avances; 1995

16. Llodra-Calvo JC, Bravo-Pérez M, Cortés-Martinicorena FJ. Encuesta de salud oral en España (2000). RCOE 2002; 7: 19-63.

17. Bravo-Pérez M, Casals-Peidró E, Cortés Martinicorena FJ, Llodra-Calvo JC. Encuesta de salud oral en Espa a 2005. RCOE 2006; 11: 409-456.

18. Cuenca E, Álvarez MT. Evolución de la salud bucodental en España, en los últimos 20 años. Arch Odontoestomatol 1991; 1: 45-51.

19. Noguerol B, Sicilia A. ¿Está disminuyendo la caries en España? Rev Actual Odontoestomatol Esp 1990; 50: 71-75.

20. FDI, OMS. Patrones cambiantes de salud buco dental e implicaciones para los recursos humanos dentales. Parte primera. Arch Odontoestomatol 1986; 2: 23-40.

21. Cortés Martinicorena FJ, Cerviño Ferradanes S, Casals Peidró E. Servicios Públicos de Salud bucodental en España. Legislación y cartera de Servicios en las CCAA $2^{\text {a }}$ Ed. Barcelona: SESPO; 2005.

22. López MA, Shukeir GS. Caries dental, su importancia en salud pública. Cient dent 2004; 1: 97-102.

23. Bravo M. Age-period-cohort analysis of dentist use in Spain from 1987 to 1997. An analysis based on the Spanish Nacional Health Interview Surveys. Eur J Oral Sci 2001; 109: 149-154

24. WHO/OMS. Global Oral Data Bank. CAPP Home Page: http://www.whocollab. od.mah.se/index. 23-06-2008 último acceso.

25. Orden PRE/2622/2007, de 7 de septiembre. «Cuadro médico de exclusiones exigible para el ingreso en los centros docentes militares de formación». Boletín Oficial de Defensa, n. ${ }^{\circ} 183$ (18-09-2007).

26. Resolución $452 / 38001 / 2008$ de 11 de enero, de la subsecretaría, por la que se convocan las pruebas selectivas para el ingreso en los centros docentes militares de formación para el acceso de nacionales y extranjeros a la condición de militar de tropa y marinería. BOE n 15 (17-01-2008).

27. Norma técnica $15 / 02$ sobre la Organización y funcionamiento de los servicios de odontología en el Ejército de Tierra (30-12-2002).

28. North Atlantic Treaty Organization. Military Agency for Standardization. MAS (ARMY) 255-MED/ 2466 (Ratification draft 1) (1 $1^{\mathrm{a}}$ edición). Dental fitness standards for military personnel and a dental fitness classification system. Unclassified (30-01-1998).

29. North Atlantic Treaty Organization. Military Agency for Standardization. MAS (ARMY)-2466 ME (Ratification draft 1) ( $2^{\mathrm{a}}$ edición). Dental fitness standards for military personnel and a dental fitness classification system. Unclassified (13-04-2006).

30. Ejército de Tierra. Mando de Adiestramiento y Doctrina. OR7-603. Orientaciones. Sanidad en Operaciones. Resolución 552/18773/03. Boletín Oficial de Defensa n. 222 (13-11-2003).

31. Richardson PS. Dental Risk Assessment for Military Personnel. Mil Med 2005; 170: 542-545.

32. Chaffin J, Marurger T, Fretwell D. Dental class 3 intercept clinic: A model for treating class 3 soldiers. Mil Med 2003; 168: 548-552.

33. Oral Health Surveys. Basic methods. 4th Ed World Health Organization; Geneva: 1997.

34. Hopcraft MS, Morgan MV. Comparison of radiographic and clinical diagnosis of approximal and occlusal dental caries in a young adult population. Community Dent Oral Epidemiol 2005; 33: 212-218.

35. Samara G, Pitta A, Lopez MA, Torres M. Prevalencia clínica y radiográfica de caries en escolares de 12, 15 y 18 años del distrito sanitario Cabula-Beirú. Salvador-Bahía. Brasil: Resultado del estudio piloto. Prof Dent 2004; 3: 161-167.

36. Segura-Egea JJ. Sensibilidad y especificidad de los métodos diagnósticos convencionales de la caries oclusal según la evidencia científica disponible. RCOE 2002; 5: 491-501. 
37. Peña-lopez JM, Carrasco-Lopez N, Fernandez-Zuizarreta JJ, Nuño-Mateo F, Villa-Vigil MA. Diferencias en el diagnóstico de caries entre la exploración clínica y radiográfica. Av Odontoestomatol 1999; 15: 303-306.

38. Pitts NB. The use of bitewing radiographs in the management of dental caries: scientific and practical considerations. Dentomaxillofac Radiol 1996; 25: 5-16.

39. Cortes FJ, Ramón R, Cuenca E, Baños S, Nevot C. Niveles de salud dental vs sobre tratamiento en estudiantes de odontología y medicina. Arch Odont-Estom Prev y Comunit 1996; 12: 395-402.

40. Rodríguez R, Castaño A, González A. Estado de la salud dental en una muestra de la población militar española. Rev Eur Odont-Estomatol 2001; 4: 195-204.

41. Hopcraft M, Morgan M. Dental caries experience in a young adult military population. Aust Dent J 2003; 48: 125-129.

42. Hopcraft MS, Morgan MV. Dental caries experience in Australian Army recruits 2002-2003. Aust Dent J 2005; 50: 16-20.

43. Senna A, Campus G, Gagliani M, Strohmenger L. Socio-economic influence on caries experience and CPITN values among a group of Italian call-up soldiers and cadets. Oral Health Prev Dent 2005; 3: 39-46.

44. Peres MA, Peres KG, Traeert J, Zabot NE, Lacera JT. Prevalence and severity of dental caries are associated with the worst socio economic conditions: A Brazilian cross-sectional study among 18-year-old males. J Adolesc Health 2005; 37 103-109.

45. Ribeiro E, Peres MAA, Marcenes W. Dental caries and socioeonomic conditions: A cross-sectional study among 18 years-old male in Florianopolis, Santa Catarina State Brasil. Rev Saúde Pública 2002; 18: 699-706.

46. Amaral MA, Nakama L, Conrado CA, Matsuo T. Dental caries in young male adults: prevalence, severity and associated factors. Braz Oral Res 2005; 19: 249-255.

47. Ceylan S, Açikel C, Okçu K, Selim K, Tekbas F, Ortakoglu K. Evaluation of the dental health of the young adult male population in Turkey. Mil Med 2004 169,11: 885-889

48. Levin L, Shenkman A. The relationship between dental caries status and oral health attitudes and behavior in young Israeli adults. J Dent Educ 2004; 68: 1185-1191.

49. Christen AG, Park PR, Graves RC, Young JM, Rahe AJ. United States Air Force survey of dental needs, 1977: methodology and summary of finding. J Am Dent Assoc 1979; 98: 726-730.

50. Keeble GB, Rugg-Gunn AJ. Dental Health of Men in the Royal Navy 1978-80. Br Dent J 1983; 155: 336-340.

51. Richardson PS, Mc Intyre IG. Dental treatment needs of a cohort of Royal Air Force recruits over 5 years. Community Dental Health 1996; 13: 11-16.

52. Sgan-Cohen HD, Horev T, Shlomo PZ, Katz J, Eldad A. The prevalence and treatment of dental caries among Israeli permanent Force Military Personnel. Mi Med 1999; 164: 562-565.

53. Horev T, Katz J, Almog D, Goldberg A, Shpigelman A, Rajnaj W. Oral health disparities between ranks in a military environment: Israel Defense Force as a model. Mil Med 2003; 168: 326-329.

54. Sgan-Cohen HD, Katz J, Horev T, Dintel A, Eldad A. Trends in caries and associated variables among young Israeli.

55. Doughan B, kassak K, Bourgeois DM. Oral helth status and treatmente needs of 35-44 years old adults in Lebanon. Int Dent J 2000; 50: 395-399.

56. Cuenca E, Puigdollers C, Lischeid G, Jover L. Estudio de la salud oral en relación a la caries en un grupo de estudiantes de estomatología. Arch Odont-Estom Prev y Comunit 1989; 1: 15-19.

57. Cortes FJ, Moreno C, Ardanza E. La salud oral de los adultos de 33-44 años de Navarra. $1^{\circ}$ parte: hallazgos clínicos. Arch Odont-Estom Prev y Comunit 1992; 1: 7-16.

58. González VL, Bullón P. Estudio epidemiológico piloto de salud oral y necesidades de tratamiento en un grupo de adultos de 35-44 años en Sevilla. Arch OdontEstom Prev y Comunit 1989; 1: 25-31.

59. Barranco-Zafra A, Llodra-Calvo JC, Bravo-Pérez M; Gálvez-Jiménez P. Prevalencia de caries y necesidades de tratamiento en los soldados españoles. Rev And Odonto-Estomatol 1997; 7: 11-14.
60. Fernández T, De la Torre J, Lozano M, Alba J. Estudio epidemiológico de la higiene buco dental en soldados de la Base de Cerro Muriano (Córdoba). Med Mil 1994; 5: 524-527.

61. Ekstrand KR, Carvalho JC, Thylstrup A. Restorative caries treatment patterns in Danish 20-year-old males in 1986 and 1991. Community Dent Oral Epidemiol 1994; $22: 75-79$

62. Ankkuriniemi O, Ainamo J. Dental health and dental treatment needs among recruits of the Finnish Defense Forces 1919-91. Acta Odontol Scand 1997; 55: 192-197.

63. Källestal C. Dental caries in 16 and 18 year old adolescents in northen Swden Scand J Dent Res 1991; 99: 100-105.

64. Asmyhr O, Grytten L, Grytten J. Trends in caries experience among male military recruits in Norway. Community Dent Oral Epidemiol 1994; 22: 206-207.

65. Asmyhr O, Nielsen J, Norheim PW. Dental health and the use of dental services among Norwegian recruits in1988. Nor Tannlaegearen Tid 1991; 101: 108-110.

66. Antoft P, Gadegaard E, Jepsen PS. Caries experience dental health behaviour and social status. A comparative study among Danish military recruits in 1972 and 1982. Community Dent Health 1988; 5: 255-264.

67. Antoft P, Rambusch E, Antoft H, Christensen HW. Caries experience, dental health behaviour and social status, three comparative surveys among Danish military recruits in 1972,1982 and 1993. Community Dent Healt 1999; 16: 8084.

68. Marker OT, Vigild M, Praetorius F. Oral health problems and treatment need in Danish military personnel recruited for United nations service. Mil Med 1997; 162: 416-421.

69. Peterson LG, Jonson G, Stadler L, Sámfors K, Gleerup A. Oral status and estimate treatment need in Swedish air force conscripts. Swed Dent J 1989;13:69-76.

70. Downer MC. The Improving Dental Health of United Kingom Adults and Prospects for the Future. Br Dent J 1991; 170: 154-158.

71. Katz J, Peretz B, Sgan-Cohen HD, Orbe T, Eldad A. Periodontal status by CPITN, and associated variables in an Israeli permanent military population. J Clin Periodontol 2000; 27: 319-324.

72. Sgan-Cohen HD, Donchin M, Goultschin J, Soskolne A, Brayer L. Periodontal treatment needs, by CPITN, among employees of a Jerusalem hospital. Int Dent J 1992; 42: 92-96.

73. Chaffin JG, Mazuji N. Class 3 dental treatment time. Mil Med 2004;169: 696-8.

74. Spencer AJ, Davies M, Slade G, Brennan D. Caries prevalence in Australasia. Int Dent J 1994; 44: 415-423.

75. Aleksejuniene J, Eriksen HM, Holst D. Variation in caries and treatment experience in 35-44-years-old Lithuanians. Community Dent Oral Epidemiol 2000; 28: 365-364

76. Julihn A, Agholme MB, Grindefjord M,Modeer T. Risk factors and risk indicators associated with high caries experience in Swedish 19-year-olds. Acta Odontol Scand 2006; 64: 267-273

77. Anónimo. Caries diagnosis and risk assessment. A review of preventive strategies and management. J Am Dent Assoc 1995; 126 Suppl: 1S-24S.

78. Fontana M, Zero DT. Assessing patients caries risk. J Am Dent Assoc 2006; 137: 1231-1239.

79. Journal of the California Dental Association. Home Page «www.cdafoundation org/journal». 13-11-2009 último acceso.

80. Mongeau SW. USAF dental readiness classifications and caries-Risk assessment. Mil Med 2008; 173: 42-47.

81. Byrappagari D, Mascarenhas AK, Chaffin JG. Association of caries and tobacco risk with dental fitness classification. Mil Med 2006; 171: 415-419.

82. Reich E, Lussi A, Newbrun E. Caries-risk assessment. Int Dent J 1999; 49: 15-26.

83. Chaffin J. Dental population health measures: supporting Army transformation. Mil Med 2003; 168: 223-226.

84. Zero D, Fontana M, Lennon AM. Clinical applications and outcomes of using indicators of risk in caries management. J Dent Educ 2001; 65: 1126-1132. 